\title{
Quand-p et contraste de polarité : étude syntaxique, sémantique et pragmasyntaxique
}

\author{
Saez Frédérique \\ Université de Toulouse II-Le Mirail/Laboratoire CLLE-ERSS \\ saez@univ-tlse2.fr
}

\section{Introduction}

\subsection{Objectif}

L'objet du présent article est de rendre compte du fonctionnement de constructions, à notre connaissance, peu étudiées du français, et que l'on trouve aussi bien à l'oral qu'à l'écrit. Il s'agit des structures dans lesquelles une proposition introduite par quand (désormais quand-p) est non régie ${ }^{1}$, et apparait en contraste de polarité avec une proposition antécédente (désormais $q^{2}$ ), contraste auquel s'ajoute un effet de surenchère que l'on peut ainsi modéliser : $q<q u a n d-p^{3}$. Ainsi dans les exemples suivants empruntés à Benzitoun (2007) :

1. Nous pataugions, quand nous ne glissions pas. (Benzitoun, $2007: 237$ )

2. Chaque Arménien perd un proche quand ce n'est pas toute sa famille (Benzitoun, $2007: 237$ )

Enoncés que nous opposerons (section 1.3 et suivantes) aux quand-p rectionnelles de polarité négative comme (3) infra :

3. J'écoutais de la musique quand je ne travaillais pas (Diakhoumpa, cité par Benzitoun, $2007: 238$ )

Benzitoun (2007) analyse conjointement les énoncés (1) \& (2). Pour notre part, nous espérons montrer que si les quand-p de (1) \& (2) sont toutes deux non-rectionnelles ( $v s$. la quand-p de (3) ainsi que nous le démontrerons section 1.3), elles ne sont pas pour autant syntaxiquement équivalentes et qu'il y aurait au moins deux types de structures contrastives introduites par quand: l'une fondée sur un contraste prédicationnel et/ou actanciel (quand ne V2(>V1) pas) et l'autre fondée exclusivement sur un contraste actanciel (quand ce n'est pas). Notre objectif est donc de distinguer ces types de constructions contrastives, non seulement des quand-p rectionnelles négatives, mais encore de mettre en évidence leurs propriétés syntaxiques et sémantiques propres. Cet objectif nous conduira nécessairement à interroger le fonctionnement temporel et le statut - au moins fonctionnel, sinon catégoriel - de quand.

\subsection{Données}

Les données sur lesquelles nous fonderons notre description, sont éssentiellement écrites et issues de nos lectures scientifiques, notamment Benzitoun (2007).

\subsection{Cadre descriptif}

La description de telles constructions ( $c f .(1) \&(2))$ nécessite l'adoption de plusieurs cadres d'analyse.

Au plan syntaxique, nous empruntons la tripartition exotaxe, épitaxe et endotaxe établie par Smessaert et al. (2005) dans le cadre de l'Approche Pronominale ${ }^{4}$. Pour distinguer entre ces niveaux, les auteurs s'appuient sur les critères suivants :

- la proportionnalité pronominale (proportionality), ou la possibilité pour un constituant de commuter avec un pronom suspensif (4a) ou assertif (4b);

- l'extraction d'un constituant (clefting) avec ou sans reprise pronominale (4c); 
- et l'ordre séquentiel (désormais OS) de l'énoncé, avec ou sans reprise pronominale (fronting and leftdislocation) (4d).

par exemple :

4. J'écoutais de la musique quand je ne travaillais pas (Diakhoumpa, cité par Benzitoun, 2007 : 238)

a. proportionnalité avec un pronom suspensif : J'écoutais de la musique quand ? - Quand je ne travaillais pas.

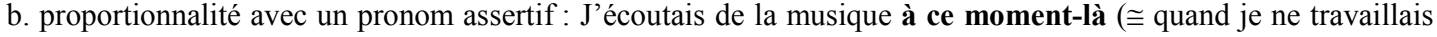
pas)

c. extraction : C'est (à ce moment-là) quand je ne travaillais pas que j'écoutais de la musique.

d. OS de l'énoncé : Quand je ne travaillais pas, j'écoutais de la musique (à ce moment-là).

L'utilisation de ces manipulations permet de mettre en évidence les contraintes qui pèsent sur les constructions et de distinguer entre trois grands niveaux d'intégration syntaxique : exotaxe, épitaxe et endotaxe. C'est une alternative concluante à la dichotomie hypotaxe vs. parataxe ${ }^{5}$ qui se révèle inadéquate lorsque l'on s'attache à décrire des phénomènes syntaxiques si fins.

Les éléments proportionnels ${ }^{6}$ à un pronom appartiennent au niveau endotaxique. Ce niveau regroupe les éléments rectionnels, et valenciels ${ }^{7}$, qui sont proportionnels à un pronom suspensif (adverbes et pronom interrogatifs tels que qui, quoi, quand) ou assertif (pronoms personnels, démonstratifs, possessifs ou indéfinis tels que ça, cela), mais aussi aux adverbes à "référenciation minimale" (minimal referential), soit les expressions du temps, du lieu et de la manière telles que à ce moment-là. Pour Smessaert et al. (2005: 476), le caractère "fini" d'un paradigme pronominal permet de rendre compte de ses combinaisons possibles avec un prédicat verbal ${ }^{8}$ :

«The possibilities or impossibility for a particular pronoun to appear in a particular paradigm is indeed meaningful: the pronouns reveal the primary or basic selectional restrictions a verbal predicator imposes on its dependents. »

Les éléments non proportionnels à un pronom suspensif ne sont pas considérés par les auteurs (2005: 478) comme relevant de l'endotaxe, mais de l'épitaxe ou de l'exotaxe. Par exemple :

5. Nous pataugions, quand nous ne glissions pas.

a. ? Nous pataugions quand ?

b. ? C'est (à ce moment-là) quand nous ne glissions pas que nous pataugions.

c. (?) Quand nous ne glissions pas, nous pataugions.

d. Quand nous ne glissions pas, nous pataugions à ce moment-là.

e. Nous pataugions à ce moment-là ( $\cong$ quand nous ne glissions pas).

La quand-p en (5) est épitaxique, car :

- elle n'est pas proportionnelle à un pronom suspensif (5a) ;

- elle ne peut être extraite dans la construction clivée (seule ou avec reprise pronominale) (5b) ;

- l'antéposition de quand-p est difficile (5c), sauf avec une reprise pronominale (5d). Nous reviendrons en section 3 sur les contraintes relatives à l'antéposition de quand- $p$;

- et elle est proportionnelle à un pronom assertif (5e) ce qui prouve qu'il y a une relation temporelle entre les deux parties de l'énoncé. Nous reviendrons sur cette relation temporelle dans la section 4.

Enfin les éléments relevant de l'exotaxe ont un ordre séquentiel fixe (antéposition obligatoire en Néerlandais), ne peuvent être extraits dans la clivée et ne sont pas proportionnels à un pronom. Nous reviendrons en section 3 sur une analyse syntaxique plus précise de ces quand-p épitaxiques. Il s'agit ici uniquement d'illustrer le cadre théorique.

Cette tripartition permet d'associer à la description syntaxique, une description pragmasyntaxique (à partir notamment des travaux de Berrendonner et [Reichler-]Béguelin (v. bibliographie)) : les niveaux épitaxique et exotaxique correspondant au niveau macrosyntaxique, et le niveau endotaxique correspondant au niveau microsyntaxique. Dans ce cadre d'analyse, les auteurs abandonnent la notion de phrase (non opératoire pour décrire une syntagmatique de type non-rectionnel) au profit de nouvelles unités linguistiques ainsi définies : la clause est l'unité maximale de la microsyntaxe. Elle est conçue par Berrendonner (2002b: 25) comme «un «îlot» de dépendances grammaticales», i.e. les unités 
morphosyntaxiques qui la composent entretiennent des relations de détermination et de solidarité (Hejlmslev, 1968) dont les contraintes peuvent être décrites distributionnellement. Les énonciations sont les unités minimales de la macrosyntaxe. Chaque énonciation est une «entité informationnelle ayant valeur d'acte de langage » (Béguelin, 2003: 113), leur fonction est donc de modifier l'état des connaissances partagées des interlocuteurs, ou mémoire discursive (M). Ainsi définies, les énonciations de clauses sont donc soumises à une contrainte d'appropriété puisqu'elles supposent un certain état antérieur de $\mathrm{M}$ à partir duquel elles peuvent opérer des révisions, transformations, ajouts... Les énonciations de clauses peuvent être regroupées en mini-programmes discursifs, appelés périodes, caractérisées par un intonème conclusif en final. Dans une visée praxéologique, les périodes, unités maximales de la macrosyntaxe, sont des programmes discursifs complets dans le sens où ils réalisent

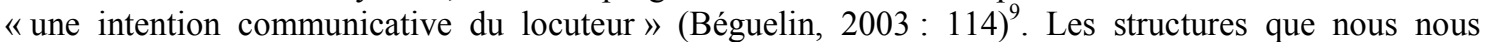
proposons d'analyser ici (ainsi de (1) et (2)) sont donc des périodes binaires ou bi-clausales AZ, ce que l'on peut ainsi représenter d'après Berrendonner (2002b: 25), « où le symbole E représente l'acte d'énoncer une clause assortie d'un intonème », noté $S$ pour « suite » (ou suspensif) et $\mathrm{F}$ pour « final» (ou conclusif) :

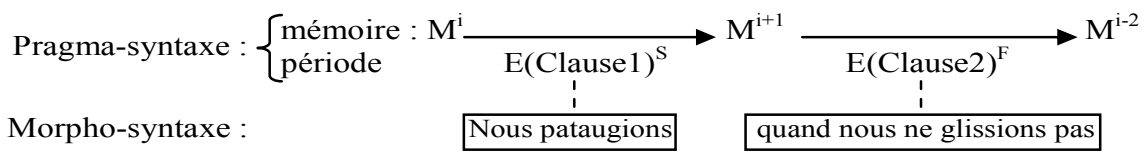

Figure 1 : représentation de la combinatoire des deux syntaxes d'après Berrendonner (2002b : 25).

Suite à cette rapide présentation du cadre descriptif, nous rappelerons brièvement les analyses antérieures proposées pour ces constructions, puis nous effecturons notre propre analyse dans le cadre que nous nous sommes fixée.

\section{Rappel critique des analyses antérieures}

Dans le cadre de l'Approche Pronominale de l'équipe du GARS (Blanche-Benveniste, et al. (1984)), Benzitoun (2007 : 237 et suiv.) propose pour ces constructions une analyse en liste paradigmatique avec contraste de polarité, et, se réclamant de Diakhoumpa (1989), il énonce une contrainte sémantique pesant sur les verbes de $q$ et $p$ : ils doivent être dans une relation de surenchère, le premier étant senti moins "fort" que le second, et effectivement on trouve les couples prédicationnels suivants :

6. Nous pataugions, quand nous ne glissions pas. (Benzitoun, $2007: 237$ ) : patauger $<$ glisser

7. En 1948, l'idée des droits de l'homme est loin d'être toute-puissante : le marxisme - qui se trouve à son apogée intellectuel dans le monde - la relativise quand il ne la récuse pas tout uniment (PRES:CourrierInt, Benzitoun, 2007 : 237) : relativiser < récuser

8. Jamais l'écart n'a été aussi énorme, aussi scandaleux, entre une minorité de possédants, dont les revenus s'accroissent d'année en année, de 20,50, voire $100 \%$ et la majorité des salariés dont les salaires, au mieux stagnent quand ils ne baissent pas du fait de la précarité (POLI:Laguiller, Benzitoun, $2007: 237$ ) : stagner < baisser

9. Ses dirigeants sont " jugés » en 1952, quand ils n'ont pas, comme le grand acteur Solomon Mikhoels, été assassinés avant. (M-Diplo, Benzitoun, $2007: 276)^{10}$ : être jugé < être assassiné

Toutefois, cette relation de surenchère peut aussi se fonder sur les objets des verbes de $q$ et $p$ :

10. Bref depuis un an, je passe minimum $40 \mathrm{~h}$ sur le net quand ça ne monte po à $120 \mathrm{~h}$, à participer à des forums, à commander mes billets de train (FORM:Multimed, Benzitoun, $2007: 238$ ) : passer 40h < monter à $120 \mathrm{~h}$

11. Aujourd'hui des malades sont renvoyés, sans soins spécifiques, dans des structures médico-sociales, quand ils ne sont pas rendus à leurs familles (POLI:PCF, Benzitoun, 2007 : 237) : renvoyés dans des structures médicosociale < rendus à leurs familles

12. Chaque Arménien perd un proche quand ce n'est pas toute sa famille (Benzitoun, 2007 : 237) : perdre un proche $<$ toute sa famille

Enfin, cette relation peut se construire sur les topoï appelés par $q$ et $p$, comme c'est le cas dans les deux derniers exemples : 
13. La richesse matérielle du capitalisme s'expose ainsi auprès de populations démunies de tout et qui, bien souvent, crèvent de faim quand ce n'est pas du paludisme ou du SIDA (Benzitoun, $2007: 240$ )

14. Il joue dans la cour de son immeuble, ou sur le parking, quand ce n'est pas dans les parties communes de l'immeuble (www.sdv.fr, Benzitoun, $2007: 240$ )

En (13) crever de faim peut apparaitre comme moins 'fort' que crever du paludisme ou du SIDA dans le sens où l'on meurt de faim mais qu'en plus de la faim, on a les maladies; et dans (14) jouer dans la cour de l'immeuble, ou le parking peut apparaitre comme moins 'fort' que jouer dans les parties communes car il est moins gênant pour le reste des habitants de l'immeuble que l'on joue à l'extérieur qu'à l'intérieur, d'où l'effet de surenchère.

L'effet de surenchère n'est donc pas réservé uniquement aux verbes de $q$ et $p$. La contrainte sémantique est donc plus large que ce que ne laisse supposer Benzitoun (2007) et porte soit sur le sémantisme des verbes (contraste prédicationnel), soit sur leurs objets (contraste actanciel objet), soit encore sur les topoï auxquels renvoient $q$ et $p$ (contraste inférentiel).

Benzitoun (2007), se réclamant toujours de Diakhoumpa (1989), ajoute les contraintes suivantes :

- sémantique : on doit pouvoir observer un effet de surenchère lié à la proximité sémantique des verbes de $p$ et $q$. En l'absence de proximité sémique, quand-p devient rectionnelle (cf. (4) supra);

- de polarité : quand-p doit être de polarité négative et l'alternance positive/négative est obligatoire faute de rendre l'énoncé inacceptable :

15. ? Les salaires, au mieux, stagnent, quand ils baissent du fait de la précarité. ou : ? Les salaires, au mieux, ne stagnent pas, quand ils ne baissent pas du fait de la précarité. (Benzitoun, 2007 : 237-238)

- $\quad$ séquentielle : quand-p est contrainte à la postposition sinon elle devient rectionnelle :

16. Quand ils ne baissent pas du fait de la précarité, les salaires, au mieux, stagnent. (Benzitoun, $2007: 238$ ) ;

- temporelle : la variation des temps des verbes de $q$ et $p$ doit se faire de façon parallèle :

17. Les salaires, au mieux, stagnaient, quand ils ne baissaient pas du fait de la précarité. (Benzitoun, $2007: 238$ )

- référentielle : les sujets des verbes de $q$ et $p$ doivent être obligatoirement coréférents, sinon quand-p devient une régie ordinaire :

18. Les salaires, au mieux, stagnent, quand la bourse ne baisse pas. (Benzitoun, 2007 : 238)

Benzitoun (2007) reconnait que ces structures présentent une véritable difficulté d'analyse. Il part de l'exemple Chaque Arménien perd un proche quand ce n'est pas toute sa famille, et promeut une analyse de quand ce n'est pas comme joncteur de liste paradigmatique, à la condition d'étendre la notion de liste paradigmatique aux constructions verbales, pour quand ne V2(>V1) pas. Il préfère cette analyse de joncteur de liste paradigmatique à une autre qui verrait dans quand ce n'est pas une construction clivée tronquée. L'analyse de quand ce n'est pas comme une clivée tronquée est motivée par la présence d'exemples tels que (19) ci-dessous, qui justifient l'hypothèse :

19. Mais comment pourrait-il en être autrement, dans des sociétés où, au nom de la rentabilité, du pragmatisme et du perfectionnement technologique, on supprime de plus en plus souvent des postes d'enseignement de la philosophie, de la littérature, de l'histoire de l'art, quand ce n'est pas la recherche fondamentale qu'on remet en cause, parce qu'elle coûte de plus en plus cher et que personne ne sait en distinguer le but? (M-Diplo, cité par Benzitoun, $2007: 244)^{11}$

La réserve de Benzitoun (2007) à l'égard d'une analyse en clivée tronquée, vient de ce que pour lui, certaines constructions en quand ce n'est pas ne se laissent pas analyser comme des clivées tronquées, ainsi de (20) : 
20. Mais l'opération inverse doit également se réaliser : les prestations à la télévision ont pour objectif de faire connaître le "produit», de créer une image, quand ce n'est pas de la corriger ou encore de la modifier totalement (Benzitoun, $2007: 244$ )

a. * quand ce n'est pas de la corriger totalement qu'elles ont pour objectif

Pour notre part, nous analysons quand ce n'est pas comme une clivée tronquée, par rapprochement avec l'exemple (19) ci-dessus dans lequel le verbe " remettre en cause » introduit une nuance sémantique par rapport au verbe antécédent, « supprimer », ce qui empêche l'ellipse de la $q u-P$. De plus, l'inacceptabilité (20a) vient de la présence d'une proposition infinitive qui ne peut en aucun cas apparaitre dans la clivée, ou la clivée tronquée. Ce test n'est donc pas un contre-argument significatif à l'analyse de quand ce n'est pas comme une clivée tronquée.

Un autre argument en faveur d'une analyse de quand ce n'est pas comme une clivée tronquée survient dans le partage du prédicat et du sujet. Nous signalons toutefois que s'il y a systématiquement partage du sujet, il n'y a pas forcément coréférence, notamment dans le cas des noms collectifs populations (ex. 13) ou encore de déterminants qui sélectionnent un élément de la classe chaque arménien (ex. 12).

Nous entendons par là que l'exemple (12) Chaque Arménien perd un proche quand ce n'est pas toute sa famille, ne signifie pas qu'un même individu ait perdu un proche ET toute sa famille, mais qu'un individu perd un proche, ET UN AUTRE, toute sa famille. Ainsi aussi de (13) La richesse matérielle du capitalisme s'expose ainsi auprès de populations démunies de tout et qui, bien souvent, crèvent de faim quand ce $n$ 'est pas du paludisme ou $d u$ SIDA, qui signifie que certaines populations - parmi celles qui sont démunies de tout - crèvent de faim, et d'autres crèvent non seulement de faim mais aussi de maladies. On peut donc ainsi paraphraser cette absence de coréférence :

21. Un arménien perd un proche, et l'autre toute sa famille.

22. Certaines populations démunies de tout crèvent de faim, et d'autres du paludisme ou du sida.

Si la coréférence n'est pas obligatoire, contrairement à ce que postule Benzitoun (2007) (cf. supra le commentaire de l'exemple (18)), il est toutefois nécessaire que les sujets possèdent un trait sémantique commun (pour (12) \& (13) il s'agit d'une sélection d'un ou plusieurs éléments au sein d'une classe sémantique, respectivement les arméniens et les populations démunies de tout), faute de modifier l'intégration syntaxique de quand-p qui devient rectionnelle (cf. (18) supra).

Outre le partage du sujet et du prédicat, un autre argument en faveur d'une analyse de quand ce n'est pas comme une clivée tronquée, vient de la présence d'une relation de type pointage ${ }^{12}$ entre le sujet de V1 et le sujet de la $q u-P$ tronquée, ce que l'on peut mettre en évidence grâce à l'anaphore par un SN défini :

23. Chaque Arménien perd un proche quand ce n'est pas toute sa famille (qu'il/l'un d'entre eux perd).

24. Ces populations démunies de tout, bien souvent, crèvent de faim quand ce n'est pas du paludisme ou du SIDA (que ces gens crèvent).

Si nous étions en présence d'un seul paradigme, ainsi que le postule Benzitoun (2007) dans son hypothèse de voir en quand ce n'est pas un joncteur de liste paradigmatique, alors la relation devrait être de l'ordre du liage (i.e. micro-syntaxique).

L'analyse de quand ce n'est pas comme une clivée tronquée induit donc nécessairement que nous sommes en présence de deux structures contrastives distinctes en quand: l'une qui produit un contraste prédicationnel : quand ne V2(>V1) pas, et l'autre qui produit un contraste actanciel : quand ce n'est pas.

En ce qui concerne la coréférence des sujets, si elle n'est pas obligatoire pour la construction quand ce $n$ 'est pas, elle ne l'est pas non plus pour quand ne V2(>V1) pas où l'on observe également la présence de déterminants qui sélectionnent des éléments de classe sémantique, ce que l'on pourrait ainsi gloser :

25. Ses dirigeants (parmi ceux qui n'ont pas été assassinés avant) sont «jugés » en 1952.

26. Aujourd'hui des malades (parmi ceux qui ne sont pas renvoyés à leurs familles) sont renvoyés, sans soins spécifiques, dans des structures médico-sociales.

Ces éléments posés, analysons maintenant ces quand-p dans le cadre decriptif défini en section 1.3 supra. 


\section{Analyse dans le cadre descriptif : arguments pour une syntaxe scalaire ?}

Au plan syntaxique, ces deux types de quand-p appartiennent au niveau épitaxique ainsi que l'ont montré les manipulations sous (5) supra et rappelées sous (27) infra, ainsi que les suivantes (28) effectuées sur quand ce n'est pas :

27. Nous pataugions, quand nous ne glissions pas.
a. ? Nous pataugions quand?
b. ? C'est (à ce moment-là) quand nous ne glissions pas que nous pataugions.
c. (?) Quand nous ne glissions pas, nous pataugions.
d. Quand nous ne glissions pas, nous pataugions à ce moment-là.
e. Nous pataugions à ce moment-là ( $\cong$ quand nous ne glissions pas).

28. Il joue dans la cour de son immeuble, quand ce n'est pas dans les parties communes.

a. ? Il joue dans la cour de son immeuble quand?

b. ? C'est (à ce moment-là) quand ce n'est pas dans les parties communes qu'il joue dans la cour de son immeuble.

c. ? Quand ce n'est pas dans les parties communes, il joue dans la cour de son immeuble.

d. ( ?) Quand ce n'est pas dans les parties communes, il joue dans la cour de son immeuble à ce moment-là

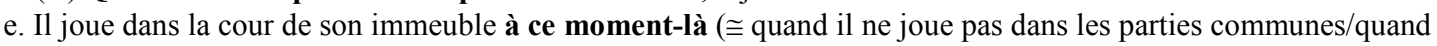
ce n'est pas dans les parties communes qu'il joue).

Ces deux structures sont bien épitaxiques, en raison de ce que :

- elles ne sont pas proportionnelles à un pronom suspensif $(27 \mathrm{a} \& 28 \mathrm{a})$;

- elles ne peuvent être extraites dans la construction clivée (seule ou avec reprise pronominale) (27b \& $28 b)$;

- l'antéposition de quand-p est difficile (27c \& 28c), sauf avec une reprise pronominale pour (27d), (28d) nous paraissant plus difficilement acceptable (nous reviendrons et expliciterons ce point dans le $\S$ suivant);

- et elles sont proportionnelles à un pronom assertif $(27 \mathrm{e} \& 28 \mathrm{e})$ ce qui prouve qu'il y a une relation temporelle entre les deux parties de l'énoncé. Nous reviendrons sur ce point dans la section 4 suivante.

En ce qui concerne la contrainte d'antéposition de quand-p, elle s'explique par la relation de pointage entre $\mathrm{E}\left(\mathrm{Clause}_{q}\right)$ et $\mathrm{E}\left(\right.$ Clause $\left.2_{\text {quand-p }}\right)$. En effet, si la première clause $\left(\mathrm{Cl}_{q}\right)$ de la période introduit le référent dans $\mathrm{M}$, alors la seconde clause $\left(\mathrm{C} 2_{\text {quand-p }}\right)$ ne saurait être antéposée à $q$ puisqu'elle présuppose le référent comme étant déjà enregistré dans $\mathrm{M}$. Toutefois, en cas de coréférence stricte des sujets $(c f$. (27) ci-dessus) alors cette antéposition devient plus acceptable. C'est pour cette raison que (27c) parait meilleure que (28c), ainsi aussi de (27d) vs. (28d). La structure quand ne V2(>V1) pas antéposée avec coréférence des sujets, introduit le référent sujet dans $\mathrm{M}$, auquel peut naturellement référer la clause qui suit, et dans laquelle on peut introduire un SN défini (27d).

En revanche, la clause introduite par quand ce n'est pas, qui dans une période $\mathrm{AZ}$ anaphorise le sujet et le prédicat de la clause antécédente (A), devient, si l'on inverse les termes de la période (ZA) (cf. (28c)), logiquement inapte à introduire dans $\mathrm{M}$ un référent dans lequel la clause qui suit puisse s'ancrer, en raison justement de ce que $\mathrm{Z}$ présuppose ce référent comme déjà enregistré dans $\mathrm{M}$. Par conséquent, même l'introduction d'une expression définie dans $q$ reste très artificielle, sinon délicate ( $c f$. . (28d)), et même en cas de simple partage sémique entre le sujet de quand-p et celui de $q$ (cf. (12) \& (13) reproduits en (29) \& (30) ci-dessous), l'antéposition de quand-p reste délicate (cf. (29a) \& (30a)) :

29. Chaque Arménien perd un proche quand ce n'est pas toute sa famille.

a. ( ?) Quand ce n'est pas toute sa famille, chaque [un autre] Arménien perd un proche à ce moment-là.

30. La richesse matérielle du capitalisme s'expose ainsi auprès de populations démunies de tout et qui, bien souvent, crèvent de faim quand ce n'est pas du paludisme ou du SIDA.

a. ( ?) Quand ce n'est pas du paludisme ou du SIDA, des populations démunies de tout [...] crèvent de faim à ce moment-là

Toutefois, l'antéposition de quand ce n'est pas est possible à condition de rétablir la qu- $P$ de la clivée tronquée, elliptique dans nos exemples et rétablie en (31) infra, et dont on trouve aussi des exemples attestés (32a vs. 32b) : 
31. Quand ce n'est pas dans les parties communes qu'il joue, il joue / [c'est] dans la cour de l'immeuble (à ce moment-là).

32. a. Quand ce n'est pas la main qui tremble, c'est l'esprit. Quand ce n'est pas l'esprit qui commande, c'est la chair. (Reverdy < Benzitoun, $2007: 245$ )

b. ? Quand ce n'est pas la main, l'esprit tremble. Quand ce n'est pas l'esprit, la chair commande.

Cette contrainte de rétablissement de la $q u-P$ tronquée en cas d'antéposition de quand ce n'est pas s'explique en ce qu'au sein de la clivée, $q u-P$ introduit un référent dans $\mathrm{M}$, qui est apte à servir de contrôleur à la clause qui suit. Ce qui n'est pas le cas dans la clivée tronquée puisqu'il y a ellipse de $q u-P$.

La coréférence des sujets semble donc accorder à quand-p une certaine liberté séquentielle, hypothèse justifiée par la prise en compte d'exemples tels que (9) \& (11), répétés en (33) \& (34) ci-dessous. En effet, dans ces énoncés on n'observe pas de coréférence stricte entre les sujets des clauses 1 et 2 mais plutôt un partage de sème. Or, en l'absence de cette coréférence, l'antéposition de quand-p seule ou avec reprise pronominale semble plus difficile (33a \& 34a), ce qui s'explique par la contrainte d'appropriété inhérente aux énonciations de clause ${ }^{13}$, et, de plus, la quand-p semble perdre sa proportionnalité au pronom assertif à ce moment-là $(33 \mathrm{~b} \& 34 \mathrm{~b})$ ce qui indique une modification dans les relations temporelles entre $q$ et quand-p, point sur lequel nous reviendrons section 4 :

33. Ses dirigeants sont jugés quand ils n'ont pas été assassinés avant.

a. ? Quand ils n'ont pas été assassinés, ses dirigeants [d'autres] sont jugés à ce moment-là.

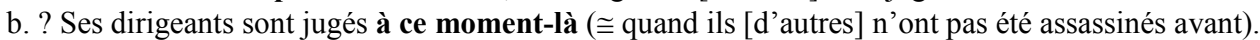

34. Aujourd'hui des malades sont renvoyés, sans soins spécifiques, dans des structures médico-sociales, quand ils ne sont pas rendus à leurs familles.

a. ? Quand ils ne sont pas rendus à leurs familles, (aujourd'hui) des malades [d'autres] sont renvoyés dans des structures médico-sociales à ce moment-là.

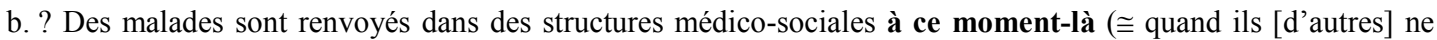
sont pas renvoyés à leurs familles).

En l'absence de coréférence, la clause quand ne V2(>V1) pas ne doit donc plus être considérée comme épitaxique mais exotaxique. L'absence de coréférence stricte modifie donc l'intégration syntaxique de quand-p, et l'OS se fige.

La coréférence des sujets semble donc bien accorder une certaine liberté séquentielle à quand-p, explicable praxéologiquement. Mais il semblerait que ce ne soit pas le seul élément qui permette l'antéposition de quand ne V2(<V1) pas. Effectivement, l'effet de renchérissement participe du figement de l'OS : en l'absence de cet effet, quand-p recouvre une certaine liberté puisqu'on trouve des énoncés tels que (35) ci-dessous, qui atténue selon nous l'acceptabilité de $(27 \mathrm{c})$ :

35. "Quand ils ne fraudèrent pas sur la qualité et le poids, les marchands se tirèrent d'affaire en vendant secrètement. » G. Lefebvre, La Révolution française. ${ }^{14}$

Pour (35), il est aisé de vérifier que la quand-p appartient elle aussi au niveau épitaxique puisque sa proportionnalité avec un pronom suspensif est difficile (36a), ainsi que son extraction dans la clivée (36b). En revanche elle nous semble, ainsi que (27) et (28), proportionnelle à un pronom assertif de type $\grave{a}$ ce moment-là (36c) :

36. a. ? Les marchands se tirèrent d'affaire en vendant secrètement quand ? - quand ils ne fraudèrent pas sur la qualité et le poids.

b. ? C'est (à ce moment-là) quand ils ne fraudèrent pas sur la qualité et le poids que les marchands se tirèrent d'affaire en vendant secrètement.

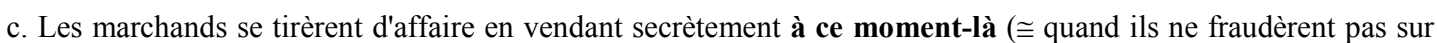
la qualité et le poids); OU : À ce moment-là ( $\cong$ quand ils ne fraudèrent pas sur la qualité et le poids) les marchands se tirèrent d'affaire en vendant secrètement

Dans (35), quand-p est antéposée, mais il n'y a pas de renchérissement entre se tirer d'affaire et frauder. En revanche, « frauder sur la qualité et le poids » peut aussi être interprété comme se tirer d'affaire: s'il n'y a donc pas de renchérissement, il y a un trait sémantique commun entre $p$ et $q$. En l'absence de se 
tirer d'affaire l'énoncé parait moins bon en maintenant l'antéposition de quand-p (cf. 37), en comparaison de (38) dans lequel quand-p est postposée :

37. ( ?) Quand ils ne fraudèrent pas sur la qualité et le poids, les marchands vendirent secrètement.

38. Les marchants vendirent secrètement, quand ils ne fraudèrent pas sur la qualité et le poids.

Si (38) parait meilleure que (37) c'est en raison du rétablissement de l'effet de renchérissement vendre secrètement $<$ frauder, inversé dans (37): frauder $>$ vendre secrètement (ce qui rejoint la nuance d'acceptabilité observée entre (27) \& (27c)).

Pour Benzitoun (2007 : 238, et (16) supra) la quand-p en (37) redeviendrait rectionnelle. Or, nous ne partageons pas ce point de vue et en effet, quand-p dans (37) \& (38) reste épitaxique ce que montre sa non proportionnalité avec un pronom suspensif (39a), ou encore sa délicate extraction dans la clivée (39b) :

39. a. ? Les marchands vendirent secrètement quand ? - quand ils ne fraudèrent pas sur la qualité et le poids

b. ? C'est (à ce moment-là) quand ils ne fraudèrent pas sur la qualité et le poids, que les marchands vendirent secrètement.

S'il nous parait délicat de considérer les quand-p de (37), (38) et même de l'exemple d'origine (35) comme rectionnelles, il n'en reste pas moins que ces énoncés sont ambigus, notamment en raison de la présence de la cataphore ils $\rightarrow$ les marchands. En effet, selon Berrendonner (1990), la cataphore relève d'une relation de liage (et non de pointage), la structure relèverait donc d'une combinatoire microsyntaxique et non macrosyntaxique. Un autre argument allant dans ce sens vient de l'impossibilité d'avoir une expression définie dans la quand- $p$ de (35) :

40. ? Quand ces gens $_{\mathbf{i}}$ ne fraudèrent pas sur la qualité et le poids, les marchands $s_{i}$ se tirèrent d'affaire en vendant secrètement.

(40) peut être acceptable à condition de ne pas considérer la coréférentialité ces gens $\leftrightarrow$ les marchands, mais alors la quand-p devient rectionnelle, ce que l'on peut vérifier par sa proportionnalité avec un pronom interrogatif (41a), et sa possible extraction dans la clivée (41b) :

41. a. Les marchands se tirèrent d'affaire en vendant secrètement quand ? - quand ces gens fraudèrent sur la qualité et le poids.

b. C'est (à ce moment-là) quand ces gens fraudèrent sur la qualité et le poids que les marchands se tirèrent d'affaire en vendant secrètement.

En (35) donc, et contrairement aux autres périodes comprenant un effet de renchérissement, la coréférence des sujets est obligatoire, puisque l'absence de coréférence (41), modifie l'intégration syntaxique de quand- $p$ qui, d'épitaxique, devient endotaxique. Ce phénomène rejoint ce que nous affirmions concernant l'antéposition de quand- $p$ et la contrainte de coréférence stricte, puisque quand- $p$ antéposée doit être capable d'introduire dans $M$ un référent, que la clause qui suit doit pouvoir présupposer.

Le tableau ci-dessous récapitule les manipulations syntaxiques effectuées précédemment, et permet de mettre en regard nos différents types de quand- $p$ selon leur degré d'intégration syntaxique :

\begin{tabular}{|c|c|c|c|c|c|}
\hline Manipulation $\quad$ Type & $\begin{array}{l}\text { Q, quand ne } \\
V 2(>V 1) \text { pas }\end{array}$ & $\begin{array}{l}\text { Q, quand ne } \\
V 2(>V 1) \text { pas }\end{array}$ & $\begin{array}{l}\text { Quand ce } \\
\text { n'est pas }\end{array}$ & $\begin{array}{l}\text { Quand ne } V \\
\text { pas, } q\end{array}$ & $\begin{array}{l}\text { Q, } \quad \text { quand-p } \\
\text { neg. }\end{array}$ \\
\hline $\begin{array}{l}\text { Proportionnalité avec: } \\
\text { - pronom suspensif } \\
\text { - pronom assertif } \\
\end{array}$ & - & $\begin{array}{l}- \\
+ \\
\end{array}$ & $\begin{array}{l}- \\
+ \\
\end{array}$ & $\begin{array}{l}- \\
+ \\
\end{array}$ & $\begin{array}{l}+ \\
+ \\
\end{array}$ \\
\hline $\begin{array}{l}\text { Extraction: } \\
\text { - sans reprise pronominale } \\
\text { - avec reprise pronominale }\end{array}$ & - & - & - & - & $\begin{array}{l}+ \\
+ \\
+\end{array}$ \\
\hline $\begin{array}{l}\text { Antéposition de quand- } p: \\
\text { - sans reprise pronominale } \\
\text { - avec reprise pronominale }\end{array}$ & - & $\begin{array}{c}- \\
(+)\end{array}$ & - & $\begin{array}{l}+ \\
+ \\
\end{array}$ & $\begin{array}{l}+ \\
+ \\
\end{array}$ \\
\hline
\end{tabular}




\begin{tabular}{|l|c|c|c|c|c|}
\hline Coréférence & - & + & \pm & + & \pm \\
\hline Niveau d'intégration & Exotaxe & \multicolumn{3}{|c|}{ Epitaxe } & Endotaxe \\
\hline
\end{tabular}

Tableau 1. Récapitulatif des propriétés syntaxiques des différents types de quand-p de polarité négative.

Il semble donc qu'il faille, pour ces périodes postuler différents sous-niveaux d'épitaxe, et, si l'on ne peut - peut-être - pas parler de syntaxe scalaire à ce stade, l'analyse syntaxique menée ici montre tout de même qu'il y a des cas intermédiaires entre épitaxe et exotaxe, et entre épitaxe et endotaxe, soit entre les degrés extrèmes d'une échelle allant de l'exotaxe (dégroupage) à l'endotaxe (coalescence ${ }^{15}$ ). Dans ce sens, les quand ne V2 $(<V 1)$ pas avec coréférence sujet et effet de surenchère seraient plus proche de l'exotaxe que les structures en quand ne $V$ pas avec coréférence sujet et maintient d'un trait sémantique commun sur les prédicats verbaux de $p$ et $q$, mais absence de renchérissement, ce que justifie le grand nombre de contraintes qui pèsent sur le type 1 par rapport au type 3 . De plus, cette hypothèse de la reconnaissance d'une syntaxe scalaire est justifiée par l'étude globale des propositions introduites par quand (cf. Saez, 2011), et il nous semble que l'examen mené ici sur quatre types de période comprenant le joncteur quand montre - à plus petite échelle - le même dynamisme qu'une étude portant globalement sur l'intégration syntaxique de quand- $p$.

En tout cas, l'analyse syntaxique de ces périodes montre qu'il faut distinguer au minimum 4 types d'intégration syntaxique de quand-p négative :

- Type 1 : exotaxique : $\{\mathrm{C} 1[q] \mathrm{C} 2[$ quand ne V2(>V1) pas] $\}$ [-coréférence sujet] (ex. 9 et 11$)$

- Type 2 : épitaxique : $\{\mathrm{C} 1[q] \mathrm{C} 2[$ quand ne V2(>V1) pas] $\}$ [+coréférence sujet] (ex. 1 à 10) $\{\mathrm{C} 1[q] \mathrm{C} 2[$ quand ce n'est pas O2(>O1)] \} [ \pm coréférence sujet] (ex. 12 à 14)

- Type 3 : épitaxique : $\{\mathrm{C} 1[$ quand ne $V$ pas] $\mathrm{C} 2[q]\}$ [+coréférence] [+trait sémantique commun aux V] (ex. 35)

- $\quad$ Type 4 : endotaxique : $\{\mathrm{C}[q$, quand ne V pas $]\}$ [ \pm coréférence] [-trait sémantique commun aux verbes] (ex. 4)

Ces périodes de type 1,2 , et 3 sont donc à la charnière de l'exotaxe et de l'épitaxe, et de l'épitaxe et de l'endotaxe, ainsi qu'à l'interface entre syntaxe et sémantique puisque la présence ou l'absence de coréférence sujet, ainsi que la présence ou l'absence de renchérissement, semblent déterminer le niveau d'intégration syntaxique de quand-p (exotaxe ou épitaxe $v s$. endotaxe).

Nous allons maintenant examiner les relations temporelles entre $p$ et $q$. Cet examen nous conduira à déterminer quelles sont les routines pragmasyntaxiques dans lesquelles s'intègrent les différentes quand-p non régies étudiées précédemment, puis nous reviendrons sur le statut de quand.

\section{Relation temporelle, rapports pragmasyntaxiques et statut de quand}

Sans le démontrer pour des raisons de place, mais en nous appuyant sur de nombreuses études antérieures (Sandfeld (1936), Chétrit (1976), Riegel et al. (2009), Maurel (1992), Muller (1996), ou encore Benzitoun (2007 ; 2008)) nous considèrerons quand comme un pronom relatif temporel indéterminé, ce qui induit, sur le modèle des relatifs que son fonctionnement soit double : (i) il comprend en lui-même un trait référentiel (ou support) temporel indéterminé (désormais [Tindet]), toujours paraphrasable par à un moment $x$. Il a donc une fonction rectionnelle dans la proposition qu'il introduit; et (ii) son statut de connecteur signale son incomplétude : il faut lui trouver dans le contexte antérieur un ancrage référentiel.

Partant de ce préalable, nous examinerons les constructions de type 1, 2 et 3 retenues dans la section précédente, soit :

- $\quad$ Type 1 : exotaxique : $\{\mathrm{C} 1[q] \mathrm{C} 2[q u a n d$ ne V2(>V1) pas $]\}$

- Type 2 : épitaxique : $\{\mathrm{C} 1[q] \mathrm{C} 2[$ quand ne V2(>V1) pas $]\}$

$\{\mathrm{C} 1[q] \mathrm{C} 2[$ quand ce n'est pas $\mathrm{O} 2(>\mathrm{O} 1)]\}$

- $\quad$ Type 3 : épitaxique : $\{\mathrm{C} 1[$ quand ne V pas $] \mathrm{C} 2[q]\}$ 
Pour le type 2, soit les structures quand ne V2(>V1) pas et quand ce n'est pas épitaxiques, $q$ semble fournir un cadre temporel à quand- $p$ dans le sens où quand anaphorise la temporalité de $q$, qui constitue de fait son contrôleur temporel ${ }^{16}$. En effet, en raison du partage de constituant entre $p$ et $q$ (coréférence des sujets), $p$ définit nécessairement la connexion établie par quand comme anaphorique, $q$ devient alors le contrôleur temporel du support temporel indéfini de quand, ce que l'on peut ainsi paraphraser :

42. Nous pataugions et, à ce moment-là, nous ne glissions pas.

43. Chaque Arménien perd un proche et, à ce moment-là, ce n'est pas toute sa famille (qu'il perd) / Il joue dans la cour de l'immeuble et, à ce moment-là ce n'est pas dans les parties communes (qu'il joue).

Mais pour le type 1, soit quand ne V2(>V1) pas exotaxique, alors la proportionnalité de quand avec et, $\grave{a}$ ce moment-là pose problème :

44. ? Ses dirigeants sont jugés et, à ce moment-là, ils n'ont pas été assassinés. / ? Des malades sont renvoyés dans des structures médico-sociales et, à ce moment-là ils ne sont pas renvoyés à leurs familles.

Enfin, pour le type 3, soit quand ne V pas, $q$, l'antéposition de quand-p interdit de voir en $q$ un contrôleur temporel possible pour quand.

Nous laissons de côté le type 4 endotaxique puisqu'il s'agit d'un cas classique de détermination temporelle de $q$ par quand- $p$. La relation temporelle est donc unidirectionnelle.

\subsection{Type 2 : quand ne V2(>V1) pas et quand ce $n$ 'est pas épitaxiques}

L'effet sémantique de la structure repose sur l'idée que chaque procès $\left(p\right.$ et $q$ ) est réalisé par le sujet ${ }^{17}$. La coréférence interdit donc la concomitance, et le rapport temporel entre $p$ et $q$ devient particulier, ce que l'on peut ainsi paraphraser :

\section{Nous pataugions quand nous ne glissions pas}

Infère : $<\grave{\mathrm{A}} \mathrm{T} 1$ nous pataugions donc à $\mathrm{T} 1$ nous ne glissions pas $><\grave{\mathrm{A}} \mathrm{T} 2$ nous glissions donc à $\mathrm{T} 2$ nous ne pataugions pas $>$

$=<$ Je patauge ET je glisse mais pas les deux en même temps $>$

46. Il joue dans la cour de l'immeuble quand ce n'est pas dans les parties communes

Infère : $<\grave{A} \mathrm{~T} 1$ il joue dans la cour donc à $\mathrm{T} 1$ il ne joue pas dans les parties communes $><\grave{A} \mathrm{~T} 2$ il joue dans les parties communes donc à $\mathrm{T} 2$ il ne joue pas dans la cour $>$

$=<\mathrm{Il}$ joue dans la cour ET dans les parties communes mais pas les deux en même temps $>$

Chaque partie de la structure comprend donc sa propre temporalité. Par conséquent, il ne s'agit pas ici de l'exclusion d'un procès par rapport à l'autre (glisser vs. patauger) ou d'un actant au profit d'un autre (cour de l'immeuble vs. parties communes) mais plutôt de l'exclusion d'une temporalité (T1) au profit d'une autre (T2). La question est de savoir d'où vient cette exclusion temporelle.

Quand exprime généralement la concomitance. Son rôle dans cette période serait de superposer les temporalités respectives de $p$ et $q$, or cette superposition est impossible (T1 vs. T2) en raison de la coréférence des sujets.

Ce fonctionnement temporel particulier de ces quand-p épitaxiques se comprend mieux si on les confronte à la quand-p endotaxique (type 4) rappelée ci-après :

47. J'écoutais de la musique quand je ne travaillais pas.

Dans (47) la glose pourrait être la suivante : $\langle\grave{\boldsymbol{A}} \boldsymbol{T}$ : soit j'écoutais de la musique, soit je travaillais $>$

Dans (47) il y a donc un véritable rapport de concomitance temporelle et à un même temps (T) deux procès s'excluent l'un l'autre (écouter de la musique vs. travailler).

Dans les périodes binaires où quand-p est épitaxique, soit quand ne V2(>V1) pas et quand ce n'est pas, avec coréférence des sujets, il y aurait bien une relation d'identité référentielle temporelle : quand aurait pour fonction (impossible) d'associer deux temporalités distinctes (T1 vs. T2) dans une relation de 
concomitance, simultanéité soutenue par le nécessaire parallélisme modal et aspectuo-temporel entre les verbes de $p$ et $q$, et la valeur anaphorique de $p$ visible dans la coréférence des actants sujets des V1 et V2.

Mais puisque la concomitance est impossible, $p$ doit nécessairement être de polarité négative ${ }^{18}$ ( $c f$. l'inacceptabilité de (48) infra), et il doit nécessairement y avoir insertion d'une inférence qui oppose les procès l'un à l'autre pour que la proposition puisse être interprétable, inférence qui s'appuie quant à elle, sur le trait sémantique commun aux verbes de $p$ et $q$ :

48. ? Nous pataugions quand nous glissions.

Toutefois, il ne s'agit pas de l'exclusion absolue de l'une ou l'autre des temporalités, mais uniquement de l'exclusion de leur simultanéité (c'est là que se situe le chiasme structurel), ce que l'on peut ainsi représenter :

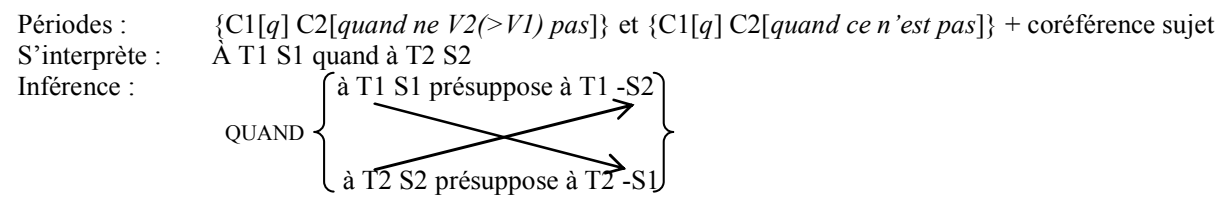

Figure 2. Interprétation temporelle des périodes binaires de type 2.

Quand aurait donc pour fonction d'unir dans un rapport d'exclusion deux temporalités distinctes. Sans cette relation d'exclusion temporelle (véhiculée par ne pas) alors la proposition est ininterprétable (48).

Il y a donc trois mouvements référentiels dans cette structure : 1. $p$ en raison de sa valeur anaphorique, spécifie que le type de connexion établie par quand est anaphorique; 2. quand se connecte donc à $q$ antécédente; et 3. $q$ représente le contrôleur temporel exclu de quand (d'où T2 -S1).

Dans ce type de période binaire, la proportionnalité de quand avec et, à ce moment-là (cf. (42) \& (43) cidessus) montre qu'il y a une relation temporelle entre $p$ et $q$, et même s'il parait délicat d'affirmer que quand-p fournit à $q$ un point d'ancrage temporel, la proportionnalité de quand-p avec à ce moment-là (cf. $27 \mathrm{~d}$,e et 28d,e ci-dessus) nous invite toutefois à interpréter une relation temporelle bidirectionnelle entre $q$ et quand-p.

En effet, nous avons dit que $q$ offrait à quand-p son contrôleur temporel. Or les deux parties de la structure ne se définissent que par la négation de l'autre, et, par conséquent, par la négation de la temporalité du procès exclu (Je patauge et je glisse mais pas les deux en même temps/il joue dans la cour et dans les parties communes mais pas les deux en même temps). Quand-p ne fournit donc pas à $q$ un point d'ancrage temporel mais la détermine temporellement en la situant par rapport à une situation (S2), durant laquelle le procès qu'elle contient, (S1), ne peut advenir (à -T2 alors S1 et à -T1 alors S2). Il s'agit donc d'une détermination temporelle de $q$, qui se fait 'par la négative', ou 'par réfection'.

En effet, si l'on se réfère à Berrendonner (2002b : 30-31), alors il est possible de voir dans cette période binaire un rapport pragmasyntaxique basé sur l'énonciation d'une clause asynaptique, qui ne présuppose aucune transformation préalable de $\mathrm{M}$, suivie d'une énonciation de clause qui peut être interprétée comme la réfection de la clause précédente qu'elle annule et remplace. Cette hypothèse expliquerait le fonctionnement temporel chiasmique de la période, et justifie la possible glose de quand par et, à ce moment-là (cf. (42) \& (43) supra). De plus, elle nous semble justifiée dans le sens où $\mathrm{E}_{\text {réfection }}$ suppose la présence dans $\mathrm{M}$ d'une clause isomorphe et que c'est justement le cas pour nos périodes binaires dans lesquelles, la première clause $\mathrm{E}_{\text {asynaptique }}$ se présente comme le prédécesseur isomorphe de quand-p, en raison du partage des actants sujets pour quand ne V2(>V1) pas et des sujet et prédicat pour quand ce $n$ 'est pas. Cet isomorphisme entre $q$ et quand-p, encore motivé par le parallélisme aspectuo-temporel des deux verbes, permet de renforcer notre hypothèse d'interpréter quand- $p$ comme la réfection de $q$ dans $\mathrm{M}$. Dans cet emploi quand- $p$ ne permet donc pas à $\mathrm{M}$ d'atteindre un autre état $\left(\mathrm{M}^{\mathrm{i}+2}\right)$, mais corrige un état antérieur de $\mathrm{M}$ auquel l'énonciation de la clause 1 permettait d'arriver $\left(\mathrm{M}^{\mathrm{i}+1}\right)$. Il convient donc de réviser ainsi notre premier schéma : 


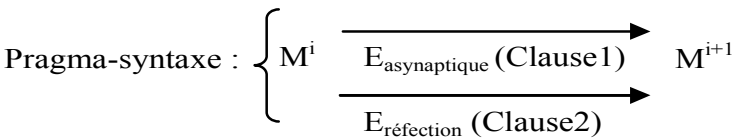

$$
\begin{aligned}
& \begin{array}{l|l|l|}
\cline { 3 - 4 } \text { Morpho-syntaxe : } \mathrm{C} 1 & \begin{array}{l}
\text { Nous pataugions } \\
\text { Il joue dans la cour de son } \\
\text { immeuble }
\end{array} & \begin{array}{l}
\text { quand nous ne glissions pas. } \\
\text { quand ce n'est pas dans les } \\
\text { parties communes }
\end{array}
\end{array}
\end{aligned}
$$

Figure 3 : représentation de la routine pragmasyntaxique des périodes binaires comprenant une quand- $p$ négative épitaxique, élaborée d'après Berrendonner (2002b : 30).

Pour quand ce n'est pas, en cas d'absence de coréférence, les choses sont un peu différentes au niveau de la relation temporelle mise en place. En effet, s'il n'y a pas de coréférence, les deux procès peuvent se dérouler dans le même temps, ce que l'on peut ainsi gloser :

49. $<$ À T1 chaque arménien perd un proche \& À T1 chaque arménien perd toute sa famille. $>$ oU $:<$ À T, un arménien perd un proche $\&$ un autre perd toute sa famille. $>$

En cas d'absence de coréférence, la clause quand ce n'est pas met en contraste les actants à la fois sujets (l'un vs. l'autre) et objets (un proche vs. toute sa famille), et la concomitance est permise.

En revanche, on retrouve pour quand ce n'est pas sans coréférence sujet, un fonctionnement équivalent pour quand: 1. $p$ en raison de sa valeur anaphorique, spécifie que le type de connexion établie par quand est anaphorique; 2. quand se connecte donc à $q$ antécédente; et 3. $q$ représente le contrôleur temporel de quand. De surcroit la proportionnalité de quand avec et, à ce moment-là (cf. (43) ci-dessus), ainsi que la proportionnalité de quand-p avec à ce moment-là (cf. (29) \& (30) ci-dessus) permet de voir une relation temporelle bidirectionnelle entre $p$ et $q$. Encore une fois, malgré l'absence de coréférence, notre

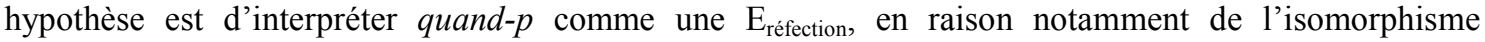
prédicationnel entre les deux clauses de la période.

\subsection{Type 1 : quand ne V2(>V1) pas exotaxique}

Ici, l'absence de coréférence autorise la concomitance, puisque par le biais du contraste entre les sujets, les deux procès peuvent se dérouler dans le même temps :

50. Aujourd'hui des malades sont renvoyés sans soins spécifiques dans des structures médico-sociales, quand ils ne sont pas rendus à leurs familles

$=<\dot{\mathbf{A}} \mathbf{T}$ (Aujourd'hui) certains malades sont renvoyés sans soins spécifiques dans des structures médico-sociales, et d'autres sont rendus à leurs familles>

Dans une intégration exotaxique, la concomitance est permise, tout autant que le décalage temporel :

51. Ses dirigeants sont jugés quand ils n'ont pas été assassinés avant.

Le renchérissement entre les verbes de $p$ et $q$, autorise à dire que la connexion établie par quand est anaphorique (V2 $>\mathrm{V} 1)$; ce qui signifie que $q$ représente le point d'ancrage temporel du support [Tindet] de quand, mais, contrairement à ce qui se produit au niveau épitaxique, la relation temporelle n'est pas bidirectionnelle : quand- $p$ ne détermine pas temporellement $q$ ( $c f$. la non proportionnalité de quand-p avec à ce moment-là : (33) \& (34) ci-dessus).

Dans cette période binaire, si la concomitance, ou le décalage temporel sont permis, et s'il n'y a pas de relation temporelle bidirectionnelle entre les deux parties de la structure, alors il est délicat d'interpréter quand-p comme une $\mathrm{E}_{\text {réfection }}$ ainsi que nous l'avons fait pour le type 2 épitaxique précédent. Ici, en nous appuyant encore sur les travaux de Berrendonner (2002b : 29-30) nous postulerons pour la quand- $p$ un

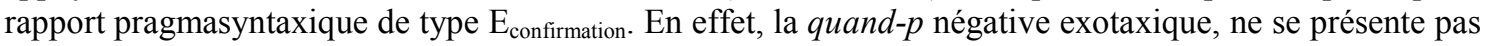
véritablement comme la continuation de ce qui précède, mais plutôt «comme un parachèvement [...], comme une action auxiliaire qui vient en expliciter après coup certains effets communicatifs. " (ibid., $2002 b$ : 30). Voici un exemple d'énonciation $\mathrm{E}_{\text {confirmation }}$ donnée par l'auteur : 


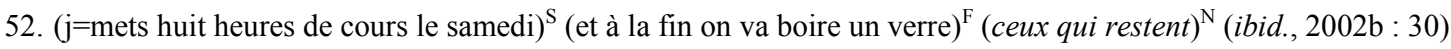

$\mathrm{Au}$ vu de (52) notre hypothèse d'interpréter la quand-p négative exotaxique comme une $\mathrm{E}_{\text {confirmation }}$ semble motivée. En effet, notre clause vient expliciter une inférence pouvant être douteuse déclenchée par l'énonciation précédente : pour (50) c'est le SN indéfinis des malades inclus dans $q$ qui est explicité par quand-p : des malades parmi ceux qui ne sont pas rendus à leurs familles (cf. la glose (26) supra); et pour (51), c'est le SN défini ses dirigeants qui est explicité par quand-p : ses dirigeants parmi ceux qui n'ont pas été assassinés avant (cf. la paraphrase (25) supra). Il ne semble donc pas tout à fait inexact de

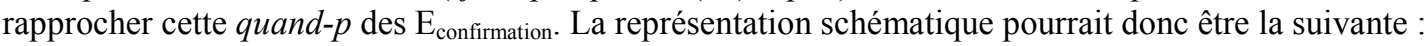

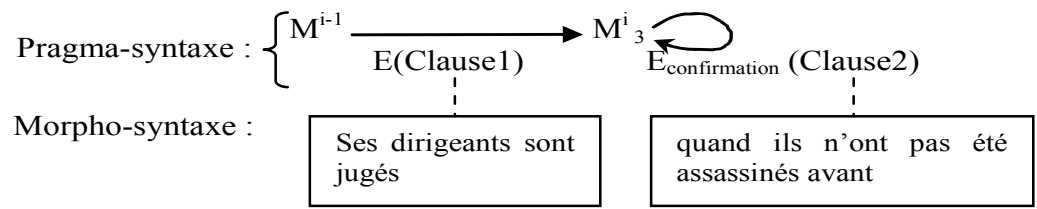

Figure 4 : représentation de la routine pragmasyntaxique de la période binaire comprenant une quand-p négative exotaxique, élaborée d'après Berrendonner (2002b : 30).

\subsection{Type 3 : quand ne $V$ pas, $q$ épitaxique}

Dans cette période binaire, quand fournit à $p$ une variable temporelle, ce qui induit que $p$ soit temporellement autodéterminée.

L'obligation de coréférence, associée à la présence d'un trait sémantique commun aux verbes de $p$ et $q$, invite à interpréter (ainsi que pour le type 2) la présence de deux temporalités distinctes, chaque procès étant réalisé par le sujet, mais pas en même temps :

53. <Ils fraudèrent sur la qualité et sur le poids ET ils se tirèrent d'affaire en vendant secrètement, mais pas les deux en même temps.>

Mais la relation cataphorique entre quand- $p$ et $q$ interdit de voir une relation bidirectionnelle entre $q$ et $p$ ( $p$ étant temporellement autodéterminée), et c'est donc quand- $p$ qui fournit à $\mathrm{q}$ un point d'ancrage temporel. Dans ce sens on se rapproche du fonctionnement temporel du type 4 endotaxique.

Toutefois, le statut épitaxique de quand- $p$ nous permet de lui attribuer un rapport pragmasyntaxique que

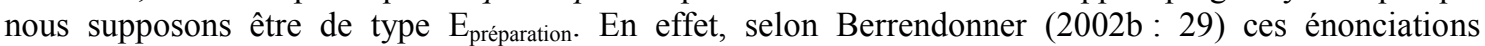
«ouvrent l'attente après elles d'une action communicative prévisible, sans laquelle elles seraient dépourvues de pertinence, et à laquelle elles servent donc de préparation. " Selon l'auteur, ces énonciations auraient pour fonction de poser «un objet-de-discours sous-déterminé » (ibid.) Voici un exemple de $\mathrm{E}_{\text {préparation }}$ donné par Berrendonner (2002b : 29) :

54. Je suis sûre d'une chose, c'est que la plupart veulent travailler [presse]

Il nous semble possible d'interpréter ainsi la quand-p épitaxique antéposée de notre exemple. En effet, son contenu informationnel relève d'un topoï $<$ les marchands sont des voleurs $>$; par conséquent qu'<ils fraudent sur la qualité et le poids $>$ n'est pas informationnellement saillant, $v s$. le contenu informationnel de $q$ qui apporte une information pertinente : <ils vendent secrètement $>$. La quand- $p$ de type 3 s'intègrerait donc dans une routine syntagmatique, ainsi modélisée par Avanzi (2005: 5): $\mathrm{E}_{\text {préparation }} \rightarrow$ $\mathrm{E}_{\text {action. }}$ Il est donc possible de représenter cette quand- $p$ épitaxique antéposée : 


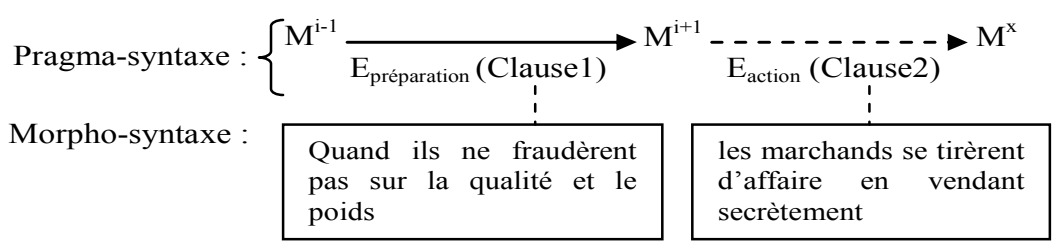

Figure 5 : représentation de la routine pragmasyntaxique de la période binaire comprenant une quand- $p$ négative épitaxique antéposée, élaborée d'après Berrendonner (2002b : 29).

\title{
4.4 Quel statut pour quand
}

Pour les quatre types de périodes étudiées (binaire et unaire), il est délicat de voir en quand un introducteur monofonctionnel. Dans les périodes binaires de type 1 et 2, respectivement exotaxique et épitaxique, son fonctionnement anaphorique le rapproche des connecteurs anaphoriques tels que définis par Berrendonner (1983). À l'opposé, son fonctionnement dans le type 4 le rapproche des relatifs à antécédent incorporé, ce que justifie sa proportionnalité avec au moment où :

55. Je travaillais au moment où je n'écoutais pas de musique

Entre les deux, on trouve le type 3 dans lequel quand fournit à $p$ une variable temporelle, et quand-p fournissant elle-même à $q$ un point d'ancrage temporel, ce que l'on peut ainsi formuler :

56. $<$ Ils ne fraudèrent pas sur la qualité et sur le poids à un moment $\mathbf{x}>_{\mathrm{i}}, \mathbf{a l o r s}_{\mathrm{i}}$ les marchands se tirèrent d'affaire en vendant secrètement.

Pour le type 3 il est délicat de déterminer exactement le statut de quand. Nous avions postulé dans des travaux antérieurs (2011), de voir dans certains emplois de quand un connecteur intégratif. La formulation, quoique peu heureuse, présentait l'avantage d'opposer le fonctionnement de quand dans le type 3, à son fonctionnement comme connecteur anaphorique (type 1 et 2), ainsi qu'à son fonctionnement de pronom relatif temporel à antécédent incorporé (type 4).

Berrendonner (2002b: 33-34) examine une série d'«opérateurs ambivalents » qui présentent les caractéristiques suivantes :

\begin{abstract}
«(i) Au plan syntaxique, ils entrent à titre de constituants dans les clauses [...]. (ii) Mais au plan sémantique, ils revêtent tous deux valeurs. L'une peut être nommée intra-clausale: elle consiste simplement en une contribution apportée au contenu propositionnel de la clause. L'autre est de nature méta-énonciative : elle consiste à commenter «sui-réferentiellement» l'énonciation de la clause et plus précisément à qualifier son rapport à l'état courant de la mémoire discursive. »
\end{abstract}

Appliquées à quand ces hypothèses permettent de clarifier sinon son statut catégoriel, au moins sa valeur sémantique. De fait, ce que nous avons nommé connecteur anaphorique ou intégratif, serait la valeur méta-énonciative de quand, qui devient alors apte à opérer des changements pragma-syntaxiques ( $c f$. les quand- $p \mathrm{E}_{\text {confirmation, }} \mathrm{E}_{\text {réfection }}$ et $\mathrm{E}_{\text {préparation }}$ ). À l'inverse, son emploi que nous avons dit être équivalent de celui des pronoms relatifs à antécédent incorporé serait sa valeur intra-clausale ( $c f$. la quand-p endotaxique).

Nous ne prétendons pas ici régler la question de la catégorisation du mot quand, toutefois, cette distinction de valeur et d'emploi fait indéniablement de quand un opérateur ambivalent, polyfonctionnel.

\section{Conclusion}

Nous espérons avoir montré que ces périodes binaires (type 1 à 3 ) sont particulièrement représentatives de l'interaction entre syntaxe, pragmasyntaxe et sémantique.

Les contraintes sémantiques sont les suivantes : 
- obligation d'une polarité négative sur quand-p;

- présence ou absence de coréférence sujet ;

- présence ou absence de l'effet de renchérissement entre V1 et V2 (ou objet O1 et objet O2) ;

- nécessaire présence d'un trait sémantique commun entre V1 et V2 ;

- ordre séquentiel.

En effet, on se rend compte que l'intégration syntaxique de quand-p semble déterminée par la présence ou l'absence de coréférence, ou la présence ou l'absence de l'effet de renchérissement. Le tableau ci-dessous représente les différents degrés d'intégration syntaxique évalués pour les quand-p négatives selon les contraintes sémantiques (nous rappelons à sa suite les quand-p concernées) :

\begin{tabular}{|c|c|c|c|c|c|c|c|}
\hline $\begin{array}{l}\text { Contraintes } \\
\text { Niveau }\end{array}$ & $\begin{array}{l}\text { Polarité } \\
\text { négative }\end{array}$ & $\begin{array}{l}\text { Coréférence } \\
\text { sujet }\end{array}$ & $\begin{array}{l}\text { Trait sémantique } \\
\text { commun }\end{array}$ & $\begin{array}{l}\text { Renchérisse } \\
\text {-ment }\end{array}$ & OS & Type de contraste & Ex. \\
\hline Exotaxe & + & - & + & $+(\mathrm{V} 1<\mathrm{V} 2)$ & Fixe & $\begin{array}{l}\text { Contraste } \\
\text { prédicationnel et } \\
\text { actanciel (sujet) }\end{array}$ & (57) \\
\hline \multirow[t]{3}{*}{ Epitaxe } & + & + & + & $+(\mathrm{V} 1<\mathrm{V} 2)$ & Fixe & $\begin{array}{l}\text { Contraste } \\
\text { prédicationnel }\end{array}$ & (58) \\
\hline & + & \pm & + & $+(\mathrm{O} 1<\mathrm{O} 2)$ & Fixe & $\begin{array}{l}\text { Contraste } \\
\text { actanciel (sujet } \\
\text { et/ou objet) ou } \\
\text { inférentiel }\end{array}$ & (59) \\
\hline & + & + & + & - & Libre & $\begin{array}{l}\text { Contraste } \\
\text { prédicationnel }\end{array}$ & (60) \\
\hline Endotaxe & \pm & \pm & - & - & Libre & Pas de contraste & (61) \\
\hline
\end{tabular}

Tableau 2 : propriétés distinctives des structures en quand-p négative selon l'intégration syntaxique.

Les exemples ci-dessous illustrent le tableau précédent :

57. Ses dirigeants sont jugés, quand ils n’ont pas été assassinés avant.

58. Nous pataugions quand nous ne glissions pas ;

59. Chaque Arménien perd un proche quand ce n'est pas toute sa famille / Il joue dans la cour de son immeuble quand ce n'est pas dans les parties communes.

60. Quand ils ne fraudèrent pas sur la qualité et le poids, les marchands se tirèrent d'affaire en vendant secrètement.

61. J'écoutais de la musique quand je ne travaillais pas.

Ce tableau nous permet d'observer que plus la quand-p est détachée du contexte antérieur (type 1 exotaxique), plus elle subit de contrainte et inversement (type 4 endotaxique).

Mais la présence ou l'absence de coréférence, ne détermine pas seulement le niveau d'intégration syntaxique, mais également la relation temporelle entre les deux parties de la structure, la relation temporelle étant elle-même en lien avec l'implication praxéologique de quand-p dans M. Le tableau cidessous représente cette interaction entre relation temporelle et coréférence (les exemples précédents fournissent l'illustration du tableau infra) :

\begin{tabular}{|c|c|c|c|c|c|}
\hline $\begin{array}{l}\text { Intégration } \\
\text { syntaxique }\end{array}$ & Ex. & Coréférence & Concomitance & $\begin{array}{c}\text { Détermination } \\
\text { temporelle }\end{array}$ & $\begin{array}{c}\text { Implication praxéologique } \\
\text { de quand-p }\end{array}$ \\
\hline Type 1: exotaxe & $(57)$ & - & \pm & $\mathrm{q} \rightarrow$ quand-p & $\mathrm{E}_{\text {confirmation }}$ \\
\hline \multirow[t]{2}{*}{ Type 2 : épitaxe } & (58) & + & - & \multirow{2}{*}{$\mathrm{q} \leftrightarrow$ quand-p } & \multirow{2}{*}{$\mathrm{E}_{\text {réfection }}$} \\
\hline & (59) & \pm & \pm & & \\
\hline Type 3 : épitaxe & $(60)$ & + & - & quand- $p \rightarrow q$ & $\mathrm{E}_{\text {préparation }}$ \\
\hline Type 4 : endotaxe & (61) & \pm & + & $q \leftarrow$ quand-p & Valeur intraclausale \\
\hline
\end{tabular}

Tableau 3 : récapitulatif des relations temporelles entre $p$ et $q$ selon l'intégration syntaxique de quand- $p$ et l'implication praxéologique de quand- $p$.

$\mathrm{Au}$ niveau épitaxique, la coréférence semble bloquer la concomitance. Inversement, au niveau exotaxique, l'absence de coréférence donne à quand-p une certaine liberté temporelle puisqu'elle peut 
être - ou non, concomitante à $q$. Au niveau endotaxique, la coréférence est libre, mais la concomitance est obligatoire. Les deux extrémités du tableau sont donc inverses (type 1 vs. type 4).

\section{Références bibliographiques}

Avanzi, M., (2007), « Regards croisés sur la notion de 'macro-syntaxe' », Tranel, 47, 39-58.

- (2005), "Quelques hypothèses à propos de la structuration interne des périodes », Proceedings of the IDP05 International Symposium on Discourse-Prosody Interfaces, C. Auran, R. Bertrand, C. Chanet, A. Colas, A. Di Cristo, C. Portes, A. Reynier \& M. Vion (éds). CD-ROM.

Béguelin, M.-J., (2003), « Variations entre macro-et mico-syntaxe : de quelques phénomènes de grammaticalisation » in Sacarano A. (a cura di), Macro-syntaxe et pragmatique. L'analyse de l'oral, Atti dell'Incontro Internationale, Dipartimento di Italianisctica, Firenze, 23-24 aprile 1999, Roma, Bulzoni Editore, 111-131.

- (2004), «Coalescences et dégroupages syntaxiques : réflexions sur les conditions pragmatiques et sémantiques des grammaticalisations». In A. Auchlin et al. (éds), Structures et discours. Mélanges offerts à Eddy Roulet. Québec : Éditions Nota Bene, 2004, 139-150, en ligne.

Benzitoun, C., (2007), Description morphosyntaxique du mot « quand » en français contemporain, Thèse de doctorat de l'université de Aix-Marseille I.

— (2008), «Qui est quand ? Essai d'analyse catégorielle », Langue Française 2|2008 n¹58, 129-143.

Berrendonner, A. et [Reichler-]Béguelin M.-J., (1989), «Décalages : les niveaux de l'analyse linguistique », Langue française 81/1, Structuration de textes: connecteurs et démarcations graphiques, 99-125, en ligne: http://www.persee.fr

- (1997), "Left dislocation in French: varieties, use and norm », in Cheshire J. \& Stein D. (eds), Taming the Verbacular: from dialect to written standard language, London \& New York, Longman, 200-217.

Berrendonner, A., (1983), « Connecteurs pragmatiques et anaphore », Cahier de linguistique française 5, 215-246, en ligne : http://clf.unige.ch/num.php?numero=5

— (1990), « Pour une macro-syntaxe », Travaux de linguistique 21, 25-36.

— (2002a), « Les deux syntaxes », Verbum 24, 23-35.

- (2002b), « Morpho-syntaxe, pragma-syntaxe et ambivalences sémantiques », in Andersen H.-L. \& Nølke H. (éds), Macro-syntaxe et macro-sémantique, Berne, Peter Lang, 23-41.

Blanche-Benveniste, C., Delofeu, J., Stefanini, J. \& Eynde, K. van den, (1984), Pronom et syntaxe. L'approche pronominale et son application au français, Paris: SELAF, pp. 245.

Chétrit, J., (1976) Syntaxe de la phrase complexe a subordonnée temporelle, étude descriptive, Paris : Klincksieck

Corminboeuf, G., (2008), L'expression de l'hypothèse en français contemporain, entre hypotaxe et parataxe. Thèse de doctorat de l'université de Neuchâtel.

Eynde, K. van den \& Blanche-Benveniste, Cl., (1978), «Syntaxe et mécanismes descriptifs : présentation de l'approche pronominale », Cahiers de Lexicologie 32, 3-27.

Hjelmslev, L., (1968), Prolégomènes à une théorie du langage, Paris, Minuit (édition originale, 1983).

Maurel, J.-P., (1992), « Subordination inverse et neutralisation du relatif. », Travaux de linguistique du CerLICO 5, $72-88$

Muller, Cl., (1996), La subordination en français, Paris : Armand Colin

Riegel, M., Pellat J.-Ch., Rioul, R., (1994, rev. 2009), Grammaire méthodique du français, Paris : P.U.F.

Saez, F., (2011), La scalarité de l'intégration syntaxique: étude syntaxique, sémantique et pragmatique de la proposition en quand, Thèse de doctorat de l'université de Toulouse 2-Le Mirail,

Sandfeld, Kr. (1965), Syntaxe du français contemporain, les propositions subordonnées, 2e éd., Genève (Suisse) : Droz S.A. 
Smessaert, H., Cornillie, B., Djivar, D., \& Eynde, K. van den, (2005), "Degrees of clause integration from endotactic to exotactic subordination”, Linguistics 43, 471-529

\begin{abstract}
${ }^{1}$ Nous entendons par non régie une proposition qui n'entretient pas de relation dépendancielle avec une autre proposition. Nous nous inscrivons dans le cadre de l'Approche Pronominale et, dans ce cadre d'analyse, la notion de rection désigne l'ensemble des éléments se trouvant dans la dépendance d'un recteur, que celui-ci soit verbal, nominal ou adjectival. Les quand-p étudiées ici ne se trouvent justement pas dans une relation dépendancielle avec le verbe qui précède, ainsi que nous le démontrerons section 1.3, et c'est pour cela que nous les qualifions de non régies
\end{abstract} ou non rectionnelles.

${ }^{2}$ Pour ne pas influer sur l'interprétation de l'une et l'autre des parties de l'énoncé, nous utiliserons les symboles $p$ (pour la proposition introduite par quand) et $q$ (pour la proposition non introduite par quand). Ici ces symboles ne sont pas révélateurs de relation syntaxique ou logique, nous souhaitons leur attribuer ici une totale neutralité.

${ }^{3}$ Dans cette formule, le symbole «<» indique l'effet de surenchère : un élément de $q$ est compris comme étant sémantiquement moins 'fort' qu'un élément de quand-p.

${ }^{4}$ Cf. Van den Eynde et al. (1978) et Cl. Blanche-Benveniste et al. (1984).

${ }^{5}$ Dans l'espace qui nous est imparti nous ne pouvons nous étendre sur cette opposition binaire, aussi nous renvoyons à nos travaux (Saez, 2011) ou encore à ceux, entre autres, de Benzitoun (2007) ou Corminboeuf (2008) qui discutent longuement de cette dichotomie.

${ }^{6}$ Smessaert et al. (2005: 478 et suiv.) distinguent entre proportionnalité interne et externe. La notion de proportionnalité interne s'applique aux locutions conjonctives comme $d u$ moment que (sens causal) et à la condition que par exemple, dont un des composants peut ou non être proportionnel à un pronom : à cette condition/ à quelle condition vs *de ce moment $/ *$ de quel moment. Elle ne s'applique donc pas aux conjonctions simples (non phrasal or simple conjunctions) comme quand.

${ }^{7}$ Parmi les éléments rectionnels, les éléments de valence sont sélectionnés par le prédicat.

${ }^{8}$ Smessaert et al. (2005: 476) parlent de «verbal construction kernel ».

${ }^{9}$ Notre présentation des travaux de l'équipe de Fribourg est ici nécessairement succincte, nous référons donc aux travaux de leurs auteurs, notamment Berrendonner \& [Reichler-]Béguelin (1989; 1997), Berrendonner (2002) et Béguelin (2003).

${ }^{10}$ Benzitoun (2007) classe cet énoncé parmi les quand-p adjointes, nous modifions donc son classement.

${ }^{11}$ Nous surlignons.

${ }^{12} C f$. Berrendonner \& [Reichler-]Béguelin (1989).

${ }^{13}$ Par contrainte d'appropriété nous entendons ici que la quand-p comprend un pronom anaphorique (ils) qui renvoie à un référent introduit par l'énonciation de clause précédente. La quand-p ne peut donc arriver avant l'énonciation de clause qui permet son ancrage référentiel dans $\mathrm{M}$.

${ }^{14}$ Cet exemple nous a été proposé par Anne Le Draoulec, lors de notre soutenance.

${ }^{15}$ Nous empruntons à Beguelin (2004) les termes de dégroupage et coalescence, à condition - pour nous - d'étendre la notion de coalescence à l'intégralité de la construction verbale (soit quand- $p$ ) et non pas au seul verbe.

${ }^{16} \mathrm{Au}$ sens de Berrendonner \& [Reichler-]Béguelin (1989), un spécificateur pour son support ou trait référentiel [Tindet].

${ }^{17}$ Contrairement à ce qui se produit si l'on remplace quand par si malgré la coréférence des sujets : je patauge si je ne glisse pas.

${ }^{18}$ On ne retrouve pas la même contrainte sur la polarité de $p$ lorsque quand-p de polarité négative est rectionnelle : J'écoutais de la musique quand je ne travaillais pas $\rightarrow$ J'écoutais de la musique quand je travaillais. 